Supplement of Biogeosciences, 11, 6417-6425, 2014

http://www.biogeosciences.net/11/6417/2014/

doi:10.5194/bg-11-6417-2014-supplement

(C) Author(s) 2014. CC Attribution 3.0 License.

(c) (i)

Supplement of

Technical Note: Linking climate change and downed woody debris decomposition across forests of the eastern United States

M. B. Russell et al.

Correspondence to: M. B. Russell (russellm@umn.edu) 


\section{Supplement Tables}

Table S1. Species groups (conifer and hardwood species) and geographic regions considered

(northern or southern region in eastern US by state) for determining differences in downed

woody debris decomposition dynamics across the eastern US.

\begin{tabular}{|c|c|c|c|c|c|}
\hline \multicolumn{6}{|c|}{ Species group } \\
\hline Conifer species & $n \dagger$ & Hardwood species & $n$ & Hardwood species & $n$ \\
\hline Abies balsamea & 527 & Acer negundo & 17 & Oxydendrum arboreum & 14 \\
\hline Abies spp. & 8 & Acer pensylvanicum & 6 & Persea borbonia & 16 \\
\hline Juniperus virginiana & 51 & Acer rubrum & 167 & Populus balsamifera & 39 \\
\hline Larix laricina & 8 & Acer saccharinum & 22 & Populus grandidentata & 44 \\
\hline Picea glauca & 28 & Acer saccharum & 113 & Populus tremuloides & 218 \\
\hline Picea mariana & 281 & Acer spp. & 29 & Populus spp. & 17 \\
\hline Picea rubens & 75 & Betula alleghaniensis & 36 & Prunus serotina & 20 \\
\hline Picea spp. & 2 & Betula lenta & 16 & Quercus alba & 84 \\
\hline Pinus banksiana & 301 & Betula papyrifera & 219 & Quercus coccinea & 13 \\
\hline Pinus echinata & 46 & Betula populifolia & 15 & Quercus ellipsoidalis & 10 \\
\hline Pinus elliottii & 36 & Betula spp. & 5 & Quercus falcata & 34 \\
\hline Pinus palustris & 11 & Carya glabra & 14 & Quercus nigra & 34 \\
\hline Pinus resinosa & 48 & Carya ovata & 14 & Quercus phellos & 9 \\
\hline Pinus strobus & 77 & Carya spp. & 24 & Quercus prinus & 40 \\
\hline Pinus taeda & 222 & Celtis spp. & 8 & Quercus rubra & 124 \\
\hline Pinus virginiana & 132 & Cornus florida & 10 & Quercus stellata & 27 \\
\hline Pinus spp. & 24 & Fagus grandifolia & 44 & Quercus velutina & 94 \\
\hline Thuja occidentalis & 184 & Fraxinus americana & 17 & Quercus spp. & 65 \\
\hline Tsuga canadensis & 13 & Fraxinus nigra & 30 & Rhizophora mangle & 18 \\
\hline Tsuga spp. & 2 & Fraxinus pennsylvanica & 13 & Robinia pseudoacacia & 15 \\
\hline Other conifer species & 62 & Fraxinus spp. & 3 & Salix nigra & 5 \\
\hline All conifer species & 2138 & Juglans spp. & 8 & Sassafras albidum & 27 \\
\hline & & Liquidambar styraciflua & 48 & Tilia americana & 21 \\
\hline & & Liriodendron tulipifera & 21 & Ulmus americana & 74 \\
\hline & & Nyssa biflora & 6 & Ulmus spp. & 5 \\
\hline & & Nyssa spp. & 6 & Other hardwood species & 259 \\
\hline & & Ostrya virginiana & 9 & All hardwood species & 2246 \\
\hline \multicolumn{6}{|c|}{ Geographic region } \\
\hline Northern US states & $n$ & Southern US states & $n$ & & \\
\hline Illinois & 39 & Alabama & 167 & & \\
\hline Indiana & 80 & Arkansas & 186 & & \\
\hline Iowa & 70 & Florida & 10 & & \\
\hline Maine & 662 & Georgia & 180 & & \\
\hline Michigan & 396 & Kentucky & 33 & & \\
\hline Minnesota & 1235 & Louisiana & 41 & & \\
\hline Missouri & 158 & North Carolina & 91 & & \\
\hline Ohio & 116 & South Carolina & 93 & & \\
\hline Pennsylvania & 100 & Tennessee & 139 & & \\
\hline Wisconsin & 373 & Virginia & 215 & & \\
\hline All northern states & 3229 & All southern states & 1155 & & \\
\hline
\end{tabular}

+ Number of observations $(n)$ 
Table S2. Ensembles of 17 climate models used.

\begin{tabular}{|c|c|c|}
\hline Model & Name & Institution (Citation) \\
\hline BCC-CSM1.1 & Beijing Climate Center-Climate System Model 1.1 & Beijing Climate Center (Xiaoge et al., 2013) \\
\hline CCSM4 & Community Climate System Model 4.0 & $\begin{array}{l}\text { National Center for Atmospheric Research } \\
\text { (Vertenstein et al., 2013) }\end{array}$ \\
\hline CESM1-CAM5 & $\begin{array}{c}\text { Community Atmosphere Model } 5.0 \text { (Community } \\
\text { Earth System Model 1.0) }\end{array}$ & $\begin{array}{l}\text { National Center for Atmospheric Research } \\
\text { (Eaton, 2013) }\end{array}$ \\
\hline CSIRO-Mk3.6 & CSIRO-Mk3.6 & $\begin{array}{c}\text { Commonwealth Scientific and Industrial } \\
\text { Research Organization (Rotstayn et al., 2012) }\end{array}$ \\
\hline FIO-ESM & $\begin{array}{c}\begin{array}{c}\text { First Institute of Oceanography-Earth System } \\
\text { Model }\end{array} \\
\end{array}$ & $\begin{array}{c}\text { First Institute of Oceanography, State Oceanic } \\
\text { Administration (Qiao et al., 2013) }\end{array}$ \\
\hline GFDL-CM3 & $\begin{array}{l}\text { NOAA Geophysical Fluid Dynamics Laboratory- } \\
\text { Climate Model } 3\end{array}$ & $\begin{array}{c}\text { National Oceanic and Atmospheric } \\
\text { Administration-Geophysical Fluid Dynamics } \\
\text { Laboratory (Griffies et al., 2011) }\end{array}$ \\
\hline GFDL-ESM2G & $\begin{array}{l}\text { NOAA Geophysical Fluid Dynamics Laboratory- } \\
\text { Earth System Model 2G }\end{array}$ & $\begin{array}{c}\text { National Oceanic and Atmospheric } \\
\text { Administration-Geophysical Fluid Dynamics } \\
\text { Laboratory (Dunne et al., 2012a; Dunne et al., } \\
\text { 2012b) }\end{array}$ \\
\hline GFDL-ESM2M & $\begin{array}{c}\text { NOAA Geophysical Fluid Dynamics Laboratory- } \\
\text { Earth System Model 2M }\end{array}$ & $\begin{array}{c}\text { National Oceanic and Atmospheric } \\
\text { Administration-Geophysical Fluid Dynamics } \\
\text { Laboratory (Dunne et al., 2012a; Dunne et al., } \\
\text { 2012b) }\end{array}$ \\
\hline GISS-E2-R & $\begin{array}{l}\text { NASA Goddard Institute for Space Studies } \\
\text { ModelE2 }\end{array}$ & $\begin{array}{c}\text { National Aeronautics and Space } \\
\text { Administration-Goddard Institute for Space } \\
\text { Studies (Schmidt et al., 2006) }\end{array}$ \\
\hline HadGEM2AO & $\begin{array}{c}\text { Hadley Centre Global Environmental Model } \\
\text { version } 2 \text { (Atmosphere-Ocean) }\end{array}$ & Met Office Hadley Centre (Martin et al., 2011) \\
\hline HadGEM2-ES & $\begin{array}{c}\text { Hadley Centre Global Environmental Model } \\
\text { version } 2 \text { (Earth System) }\end{array}$ & Met Office Hadley Centre (Martin et al., 2011) \\
\hline IPSL-CM5A-LR & $\begin{array}{l}\text { Institut Pierre Simon Laplace Climate Model for } \\
\text { the } 5^{\text {th }} \text { IPCC Report (Low Resolution) }\end{array}$ & $\begin{array}{c}\text { Institut Pierre Simon Laplace Climate } \\
\text { Modelling Centre (Mignot and Bony, 2013) }\end{array}$ \\
\hline MIROC5 & $\begin{array}{l}\text { Model for Interdisciplinary Research on Climate } \\
\text { Version } 5\end{array}$ & $\begin{array}{c}\text { Atmosphere and Ocean Research Institute } \\
\text { (University of Tokyo), National Institute for } \\
\text { Environmental Studies, and Japan Agency for } \\
\text { Marine-Earth Science and Technology } \\
\text { (Watanabe et al., 2010) }\end{array}$ \\
\hline MIROC-ESM-CHEM & $\begin{array}{l}\text { Model for Interdisciplinary Research on Climate } \\
\text { Earth System Model (Atmospheric Chemistry) }\end{array}$ & $\begin{array}{l}\text { Japan Agency for Marine-Earth Science and } \\
\text { Technology, Atmosphere and Ocean Research } \\
\text { Institute (University of Tokyo), and National } \\
\text { Institute for Environmental Studies (Watanabe } \\
\text { et al., 2011) }\end{array}$ \\
\hline MIROC-ESM & $\begin{array}{c}\text { Model for Interdisciplinary Research on Climate } \\
\text { Earth System Model }\end{array}$ & $\begin{array}{l}\text { Japan Agency for Marine-Earth Science and } \\
\text { Technology, Atmosphere and Ocean Research } \\
\text { Institute (University of Tokyo), and National } \\
\text { Institute for Environmental Studies (Watanabe } \\
\text { et al., 2011) }\end{array}$ \\
\hline MRI-CGCM3 & $\begin{array}{c}\text { Meteorological Research Institute Chemistry } \\
\text { Global Climate Model } 3 \\
\end{array}$ & $\begin{array}{l}\text { Meteorological Research Institute (Yukimoto et } \\
\text { al., 2012) }\end{array}$ \\
\hline NorESM1-M & Norwegian Earth System Model 1 & $\begin{array}{c}\text { Norwegian Climate Center (Bentsen et al., } \\
\text { 2012; Iversen et al., 2013) }\end{array}$ \\
\hline
\end{tabular}




\section{Supplement References}

Bentsen, M., Bethke, I., Debernard, J. B., Iversen, T., Kirkevåg, A., Seland, Ø., Drange, H., Roelandt, C., Seierstad, I. A., Hoose, C., and Kristjánsson, J. E.: The Norwegian Earth System Model, NorESM1-M - Part 1: Description and basic evaluation, Geosci. Model Dev. Discuss., 5, 2843-2931, 2012.

Dunne, J. P., John, J. G., Adcroft, A. J., Griffies, S. M., Hallberg, R. W., Shevliakova, E., Stouffer, R. J., Cooke, W., Dunne, K. A., Harrison, M. J., Krasting, J. P., Malyshev, S. L., Milly, P. C. D., Phillipps, P. J., Sentman, L. T., Samuels, B. L., Spelman, M. J., Winton, M., Wittenberg, A. T., and Zadeh, N.: GFDL’s ESM2 Global Coupled Climate-Carbon Earth System Models. Part I: physical formulation and baseline simulation characteristics, J. Climate, 25, 6646-6665, 2012a.

Dunne, J. P., John, J. G., Shevliakova, E., Stouffer, R. J., Krasting, J. P., Malyshev, S. L., Milly, P. C. D., Sentman, L. T., Adcroft, A. J., Cooke, W., Dunne, K. A., Griffies, S. M., Hallberg, R. W., Harrison, M. J., Levy, H., Wittenberg, A. T., Phillips, P. J., and Zadeh, N.: GFDL's ESM2 Global Coupled Climate-Carbon Earth System Models. Part II: carbon system formulation and baseline simulation characteristics, J. Climate, 26, 2247-2267, 2012b.

Eaton, B.: User's guide to the Community Atmosphere Model CAM-5.0. National Center for Atmospheric Research. 28 pp, 2013.

Griffies, S., Winton, M., Donner, L., Horowitz, L., Downes, S., Farneti, R., Gnanadesikan, A., Hurlin, W., Lee, H.-C., Liang, Z., Palter, J., Samuels, B., Wittenberg, A., Wyman, B., Yin, J., and Zadeh, N.: The GFDL CM3 Coupled Climate Model: characteristics of the ocean and sea ice simulations, J. Climate, 24, 3520-3544, 2011.

Iversen, T., Bentsen, M., Bethke, I., Debernard, J. B., Kirkevåg, A., Seland, Ø., Drange, H., Kristjansson, J. E., Medhaug, I., Sand, M., and Seierstad, I. A.: The Norwegian Earth System Model, NorESM1-M - Part 2: Climate response and scenario projections, Geosci. Model Dev., 6, 389-415, 2013.

Martin, G. M., Bellouin, N., Collins, W. J., Culverwell, I. D., Halloran, P. R., Hardiman, S. C., Hinton, T. J., Jones, C. D., McDonald, R. E., McLaren, A. J., O'Connor, F. M., Roberts, M. J., Rodriguez, J. M., Woodward, S., Best, M. J., Brooks, M. E., Brown, A. R., Butchart, N., Dearden, C., Derbyshire, S. H., Dharssi, I., Doutriaux-Boucher, M., Edwards, J. M., Falloon, P. D., Gedney, N., Gray, L. J., Hewitt, H. T., Hobson, M., Huddleston, M. R., Hughes, J., Ineson, S., Ingram, W. J., James, P. M., Johns, T. C., Johnson, C. E., Jones, A., Jones, C. P., Joshi, M. M., Keen, A. B., Liddicoat, S., Lock, A. P., Maidens, A. V., Manners, J. C., Milton, S. F., Rae, J. G. L., Ridley, J. K., Sellar, A., Senior, C. A., Totterdell, I. J., Verhoef, A., Vidale, P. L., and Wiltshire, A.: The HadGEM2 family of Met Office Unified Model climate configurations, Geosci. Model Dev., 4, 723-757, 2011.

Mignot, J. and Bony, S.: Presentation and analysis of the IPSL and CNRM climate models used in CMIP5, Clim. Dynam., 40, 2089-2089, 2013. 
Qiao, F., Song, Z., Bao, Y., Song, Y., Shu, Q., Huang, C., and Zhao, W.: Development and evaluation of an Earth System Model with surface gravity waves, Journal of Geophysical Research: Oceans, 118, 4514-4524, 2013.

Rotstayn, L. D., Jeffrey, S. J., Collier, M. A., Dravitzki, S. M., Hirst, A. C., Syktus, J. I., and Wong, K. K.: Aerosol- and greenhouse gas-induced changes in summer rainfall and circulation in the Australasian region: a study using single-forcing climate simulations, Atmos. Chem. Phys., 12, 6377-6404, 2012.

Schmidt, G. A., Ruedy, R., Hansen, J. E., Aleinov, I., Bell, N., Bauer, M., Bauer, S., Cairns, B., Canuto, V., Cheng, Y., Del Genio, A., Faluvegi, G., Friend, A. D., Hall, T. M., Hu, Y., Kelley, M., Kiang, N. Y., Koch, D., Lacis, A. A., Lerner, J., Lo, K. K., Miller, R. L., Nazarenko, L., Oinas, V., Perlwitz, J., Perlwitz, J., Rind, D., Romanou, A., Russell, G. L., Sato, M., Shindell, D. T., Stone, P. H., Sun, S., Tausnev, N., Thresher, D., and Yao, M.-S.: Present-day atmospheric simulations using GISS ModelE: comparison to in situ, satellite, and reanalysis data, J. Climate, 19, 153-192, 2006.

Vertenstein, M., Tony Craig, T., Middleton, A., Feddema, D., and Fischer, C.: CCSM4.0 user's guide. National Center for Atmospheric Research, 126 pp 2013.

Watanabe, M., Suzuki, T., O’ishi, R., Komuro, Y., Watanabe, S., Emori, S., Takemura, T., Chikira, M., Ogura, T., Sekiguchi, M., Takata, K., Yamazaki, D., Yokohata, T., Nozawa, T., Hasumi, H., Tatebe, H., and Kimoto, M.: Improved Climate Simulation by MIROC5: Mean States, Variability, and Climate Sensitivity, J. Climate, 23, 6312-6335, 2010.

Watanabe, S., Hajima, T., Sudo, K., Nagashima, T., Takemura, T., Okajima, H., Nozawa, T., Kawase, H., Abe, M., Yokohata, T., Ise, T., Sato, H., Kato, E., Takata, K., Emori, S., and Kawamiya, M.: MIROC-ESM: model description and basic results of CMIP5-20c3m experiments, Geosci. Model Dev. Discuss., 4, 1063-1128, 2011.

Xiaoge, X., Tongwen, W., and Jie, Z.: Introduction of CMIP5 experiments carried out with the climate system models of Beijing climate center, Advances in Climate Change Research, 4, 4149, 2013.

Yukimoto, S., Adachi, Y., Hosaka, M., Sakami, T., Yoshimura, H., Hirabara, M., Tanaka, T. Y., Shindo, E., Tsujino, H., Deushi, M., Mizuta, R., Yabu, S., Obata, A., Nakano, H., Koshiro, T., Ose, T., and Kitoh, A.: A new global climate model of the Meteorological Research Institute: MRI-CGCM3 model description and basic performance, Journal of the Meteorological Society of Japan. Ser. II, 90A, 23-64, 2012. 